\title{
Wavelet-Based Harmonics Decomposition of Ultrasonic Signal in Assessment of Plastic Strain in Aluminum
}

\author{
Amir Mostavi, Negar Kamali, Niloofar Tehrani, Sheng-Wei Chi, Didem Ozevin, and \\ J. Ernesto Indacochea
}

\begin{abstract}
In nonlinear ultrasonics, the correlation between microstructural change and ultrasonic properties is investigated by the acoustic nonlinearity parameter, calculated by experimentally measuring the first and second harmonic amplitudes of ultrasound signals. The most prevalent signal processing method is to transform the time-domain signal into the frequency domain and acquire the amplitudes of each frequency from the frequency spectrum. However, the major drawback of this approach is that temporal information is not preserved and the transformation errors increase dramatically in analyzing nonlinear signals with discontinuities. In this study, two wavelet-based algorithms are introduced to analyze the waveform in nonlinear ultrasonic testing. The algorithms are applied to correlate the acoustic nonlinearity parameter and the plastic deformation of aluminum 1100 specimens, for the purpose of validation. The results showed that the acoustic nonlinearity parameter calculated through the proposed algorithms is not influenced by the signal processing variables, and the signal processing error is reduced when the waveletbased decomposition is applied.
\end{abstract}

\section{Introduction}

Ultrasonic testing (UT) is a well-established nondestructive evaluation (NDE) method that measures the material state by monitoring the propagation of high frequency elastic waves within the material. The method has been developed in many applications to detect the presence of various flaws (e.g., crack, corrosion, delamination) in materials [1-3]. The requirement that the wavelength of ultrasonic signal should be smaller than the size of defects when the linear UT is implemented limits the capability of assessing material conditions involving microstructural defects, such as early stages of fatigue and creep damage. On the other hand, on account of the advancement of UT instrumentation and the theory of nonlinear wave propagation, the nonlinear 
characteristics of the wave motion has been utilized to investigate microstructural defects [4-7]. These methods commonly rely on observing tiny changes of the elastic wave motion in the frequency and/or time domain. Consequently, the error tolerance in the nonlinear UT methods, from both measurements and signal processing, is more stringent than that in the linear UT counterparts. This paper presents a novel wavelet-based technique aiming to better extract frequency components in the nonlinear UT methods and ultimately better assess the material state due to micro-defects or damage.

Using ultrasonic testing, the material nonlinearity can be measured by two means: the stressdependent ultrasonic wave speed known as acoustoelasticity [8-11] and the detection of higher harmonics [12-15]. The acoustoelastic effect is negligibly small when the stress level is far below the yield point, which is beyond the scope of this paper. When a single-frequency (i.e., the excitation frequency) elastic wave propagates in a material, the interaction of the singlefrequency wave mode with microstructural defects generates higher-frequency components called higher harmonics. The excitation frequency is referred to in the literature as the fundamental wave frequency and the higher harmonics are integer multiples of the excitation frequency. In linear ultrasonics, the transmitting and receiving transducers are both tuned to the same frequency; however, in nonlinear ultrasonics, the receiving transducer is tuned to the second or third harmonic frequency of the transmitting transducer in order to detect the weak inherent physical nonlinearity due to the microstructural defects. Additionally, in linear ultrasonics, the excitation signal is typically a pulse signal, while a sinusoidal signal is selected in nonlinear ultrasonics in order to have a narrower bandwidth of frequency in the solution. By monitoring the amplitudes of higher harmonics or mixing two distinct incident wave frequencies to produce frequency sidebands, the nonlinearity can be quantified and correlated with material damage. The most common measurement of the nonlinearity is based on measuring the acoustic nonlinearity parameter $\beta$, which is proportional to $A_{2} / A_{1}^{2}$, the ratio of the second harmonic amplitude $A_{2}$ to the square of the first harmonic (fundamental frequency) amplitude $A_{1}[16,17]$. The solution of the nonlinear wave equation resulting from the summation of the first and second harmonic waves is presented in section 2. To date, there are several applications of nonlinear ultrasonics to assess microstructural changes in metallic alloys, such as fatigue damage [18,19], creep damage [20-22], radiation damage [15], thermal aging [23,24], and cold work [25]. 
Various wave types can be utilized for detecting microstructural damage using nonlinear ultrasonics such as longitudinal waves [22,26,27], Rayleigh waves [15,28,29], or Lamb waves [19]. Matlack et al. have presented a comprehensive review of the second harmonic generation method for detecting microstructural damage [15].

To extract the amplitudes of the fundamental and second harmonic frequencies, the most common signal processing method is to transform the time-domain signal into the frequency domain by FFT, and read the amplitude of each frequency from the frequency spectrum $[20,28,30,31]$. The major drawbacks of this approach are that temporal information is not preserved and that the transformation is ineffective in dealing with truncated signals or ones with discontinuity. Pruell et al. [19] applied the short time Fourier transform (STFT) to obtain timefrequency images. However, STFT has a fixed window size and it cannot yield good resolution based on time and frequency simultaneously. Kim and Kim [30] compared STFT and wavelet transformation (WT) and discovered that the WT is a promising method to analyze the acoustic signals. In general, the acoustic nonlinearity parameter $\beta$ rises with an increase in the density of microscopic heterogeneities, e.g., dislocation density, precipitates, or porosity. However, significant variations in the reported data and high errors in repeated measurements require more robust signal processing tools to decompose harmonic frequencies.

In this paper, we introduce a wavelet-based signal decomposition method to better extract higher harmonics from time history signals and thus more accurately obtain the acoustic nonlinearity parameter. The method in conjunction with nonlinear ultrasound testing has broad applications in assessments of material state due to micro-defects and damages [20-29]. For validation purposes, we use the proposed method to determine the variation of the acoustic nonlinearity parameter due to plastic deformation. In order to reduce the complexities in the experiment due to microstructure and the type of loading, a single-phase material, aluminum 1100 strained under uniaxial loading, has been used. The issue stemming from the contact condition of the ultrasound transducer was first addressed to minimize experimental errors. The fast Fourier transform (FFT) was also used to acquire the acoustic nonlinearity parameter. The influences of the sampling rate, frequency range, and the duration of the time-domain response on the result of frequency spectrum were all examined. The effectiveness of wavelet-based schemes was compared with the FFT. 
The organization of the paper is as follows. The relevant theory of nonlinear wave propagation used in this study is reviewed in Section 2. The signal processing techniques based on Fourier and wavelet transforms for extracting harmonic signals are discussed in Section 3. The preparation of specimens, experimental setting and results are presented in Section 4. Discussions and conclusions are presented in Section 5.

\section{Review of Nonlinear Ultrasonics}

The wave motion in solids is governed by the following equation:

$$
\frac{\rho D \mathbf{v}}{D t}=\nabla \cdot \boldsymbol{\sigma}
$$

where $\rho$ is the material density, $\mathbf{v}$ is the particle velocity, $D$ denotes the material time derivative, and the body force is neglected. $\boldsymbol{\sigma}$ is the Cauchy stress tensor and can be obtained from the strain energy density function by $\boldsymbol{\sigma}=J^{-1} \mathbf{F} \frac{\partial W}{\partial \mathbf{E}} \mathbf{F}^{T}$, where $\mathbf{F}$ is the deformation gradient and $J=\operatorname{det}(\mathbf{F})$. Under finite deformation, the general strain energy density function has the following expression:

$$
W=\frac{1}{2 !} C_{i j k l} E_{i j} E_{k l}+\frac{1}{3 !} G_{i j k l m n} E_{i j} E_{k l} E_{m n}+\ldots
$$

where $\mathbf{E}$ is the Green strain tensor and $\mathbf{C}$ and $\mathbf{G}$ are the second- and third-order elastic modulus tensors. Considering the longitudinal wave, $u$, with a sinusoidal excitation $u(0, t)=u_{0} \sin \omega t$, the solution of equation (1) is given as follows [32]:

$$
u(x, t)=u_{0} \sin \omega\left(t-\frac{x}{c_{l}}\right)+\frac{\beta^{\prime}}{4}\left(\frac{\omega}{c_{l}}\right)^{2} u_{0}^{2} x \cos 2 \omega\left(t-\frac{x}{c_{l}}\right)+\frac{\beta^{\prime 2}}{8}\left(\frac{\omega}{c_{l}}\right)^{4} u_{0}^{3} x^{2} \sin 3 \omega\left(t-\frac{x}{c_{l}}\right)+\ldots
$$

where $c_{l}$ is the propagation speed of longitudinal wave. Let $A_{1}$ and $A_{2}$ be the fundamental and second harmonic amplitudes of the above signal while converted to the frequency domain. The second harmonic is induced by the material nonlinearity and the acoustic nonlinearity parameter $\beta$ can be quantified by the following relationship: 


$$
\beta \equiv \frac{A_{2}}{A_{1}^{2}}=\beta^{\prime} \frac{x k^{2}}{4}
$$

where $k=\omega / c_{l}$ is the wave number of the fundamental wave. Note that the error of $\beta$ is sensitive to the values of $A_{1}$ and $A_{2}$, considering $x$ and $k$ are constant. The sensitivity analysis shows that the error of the calculated $\beta, \frac{A_{2} \pm \varepsilon}{\left(A_{1} \pm \varepsilon\right)^{2}}$, from equation (4) is in the order of $O\left(2 \frac{\varepsilon}{A_{1}}+\frac{\varepsilon}{A_{2}}\right)$, where $\varepsilon$ is the error from signal processing of $A_{1}$ and $A_{2}$. The error of the calculated $\beta$ is amplified when $A_{2}$ is much smaller than $A_{1}$, which is typically the case as the amplitude of the second harmonic frequency due to the microstructural damage is weak. Therefore, it is imperative that the amplitudes of $A_{1}$ and $A_{2}$ for nonlinear UT techniques be accurately measured and extracted.

\section{Signal Processing Techniques for Wave Harmonics Separation}

In this section, the basic theories and equations of commonly used methods for frequency decomposition, FFT and WT, are first reviewed to illustrate their advantages and disadvantages in the extraction of higher harmonics. Two proposed WT-based schemes are then introduced for extracting frequency amplitudes for calculation of the acoustics nonlinearity parameter. The accuracy of the proposed WT-based schemes is verified with an analytical solution and the effectiveness is compared with that of the FFT-based signal processing method.

\subsection{Fast Fourier Transform}

FFT is the most commonly used signal processing tool to find the frequency content of transient signals based on the Fourier series expansion. The Fourier series of a time-dependent signal $h(t)$ within the limit of $-T<t<T$ is:

$$
h(t)=\sum_{n=-\infty}^{\infty} c_{n} e^{\frac{i \pi n}{T} t}
$$




$$
c_{n}=\frac{1}{2 T} \int_{-T}^{T} h(t) e^{\frac{-i \pi n}{T} t} d t
$$

where $c_{n}$ corresponds to the $n^{\text {th }}$ coefficient in the Fourier series. To process a signal with finite discrete values, discrete Fourier transform (DFT) is used. In DFT, the sequence $h_{n}$ with $N$ values is transformed to the frequency domain as:

$$
H_{k}=\sum_{n=0}^{N-1} h_{n} e^{-i 2 \pi n k / N}, k=0, \ldots, N-1
$$

where $k$ is the wave number and $H_{k}$ is the corresponding sequence in the frequency domain. Due to the large computational cost of calculating DFT for large $N$, this method is seldom used. Instead, by rearranging some multiplications and sums, a simple yet effective algorithm called FFT is used which is an efficient method to compute the Fourier transform. FFT decreases the computational cost by reducing $N$, the number of points needed for computation from $2 N^{2}$ to $2 N \log N$. If the frequency is constant in time, FFT works effectively [33]. However, in nonlinear ultrasonic testing, the objective is to find the complex nonstationary higher-order harmonic signals, which potentially poses a challenge for FFT.

Moreover, some inherent characteristics of FFT affect the accuracy of signal decomposition. FFT uses global basis functions, and any perturbations in the transient signal in the time domain can dramatically affect the frequency spectrum [34]. Therefore, FFT is less accurate to handle local discontinuity in the time-varying signal with transient properties [35]. To solve this problem, Dennis Gabor introduced the STFT method to embed the temporal information into the frequency-domain analysis [36]. In this method a fixed length analysis window is introduced that slides through the time axis and computes the time-localized Fourier transform [37]. While the STFT approach is introduced to overcome the limitation of FFT that the temporal information is excluded, in this method, an implicit assumption is that the signal within the processing frame is repetitive and that the signal can only be sampled for a limited time [38]. In STFT, the time resolution can improve by decreasing the window size to calculate FFT, but the frequency resolution is reduced when the FFT window has limited data points [39]; thus STFT cannot provide good resolution in time and frequency simultaneously [35,37,40]. In conclusion, FFT is 
ineffective to decompose non-stationary transient signal accurately, and it is important to apply a more robust signal decomposition approach to extract the acoustic nonlinearity parameter.

\subsection{Wavelet Transform}

In contrast to FFT, WT uses functions that are localized in both real and Fourier spaces, called wavelets [41]. By reconstructing signals into the mother wavelets, $\psi(t)$, frequency components with the window of each wavelet can be identified. As discussed before, STFT requires a constant window length (called 'window size'), which slides through the time axis to calculate the FFT in each window and to add the temporal information of the signal into FFT [37]. Unlike the STFT method, the window size in WT is not constant, and it is a function of frequency. In higher-frequency components, the window size becomes smaller to maintain higher frequency resolution while in signals with lower frequency, higher frequency resolution is obtained by a larger window size [40]. WT has been successfully applied in obtaining time-frequency images in both linear [42] and nonlinear [43] systems and other applications in signal/image processing and damage detection [41-46]. It has also been developed in the form of Discrete Wavelet Transform, Fast Wavelet Transform, and Continuous Wavelet Transform [47].

The fundamental equation of wavelet transform can be expressed as:

$$
w_{n}(s, \tau)=\int_{-\infty}^{\infty} h(t) \psi_{s, \tau}^{*} d t
$$

where $h(t)$ is the time-domain signal, $\left({ }^{*}\right)$ denotes the complex conjugate, and $\psi_{s, \tau}$ is called the daughter wavelet and can be characterized with the dilation and translation parameters, $\mathrm{s}$ and $\tau$, respectively, as:

$$
\psi_{s, \tau}(t)=\frac{1}{\sqrt{s}} \psi\left(\frac{t-\tau}{s}\right)
$$

The dilation and translation parameters, $\mathrm{s}$ and $\tau$, vary continuously to represent different times in the time-domain signal and different contractions and dilations of the mother wavelet. The wavelet function should have zero mean and be localized in both time and frequency [48].

Additionally, the mother wavelets $\psi(t)$ must satisfy the following admissibility condition: 


$$
\int_{-\infty}^{\infty} \frac{|\hat{\psi}(\omega)|^{2}}{|\omega|} d \omega<\infty, \hat{\psi}(\omega)=\int \psi(t) e^{-i \omega t} d t
$$

Among many wavelets, the Morlet wavelet has been shown to have the best temporal and spatial resolutions [46]. In this study, the complex Morlet wavelet obtained by the product of a complex exponential and a Gaussian function is selected as the mother wavelet. The exponential decay in complex Morlet results in very precise time localization. This wavelet provides the best resolution in time and frequency; therefore, it is the most suitable wavelet for spectrogram analysis $[49,50]$. The complex Morlet wavelet has the following forms in the time and frequency domains [51]:

$$
\begin{gathered}
\psi_{M}(t)=\frac{1}{\sqrt{\pi \omega_{b}}} e^{i 2 \pi \omega_{c} t} e^{-\frac{t^{2}}{\omega_{b}}} \\
\psi_{M}(\omega)=e^{\pi^{2} \omega_{b}\left(\omega-\omega_{c}\right)^{2}}
\end{gathered}
$$

where $\omega_{b}$ and $\omega_{c}$ are the parameters controlling the frequency bandwidth and the central frequency, respectively. Figure 1 shows the complex Morlet wavelet with the central frequency of $1.5 \mathrm{~Hz}$ and bandwidth of $1 \mathrm{~Hz}$.
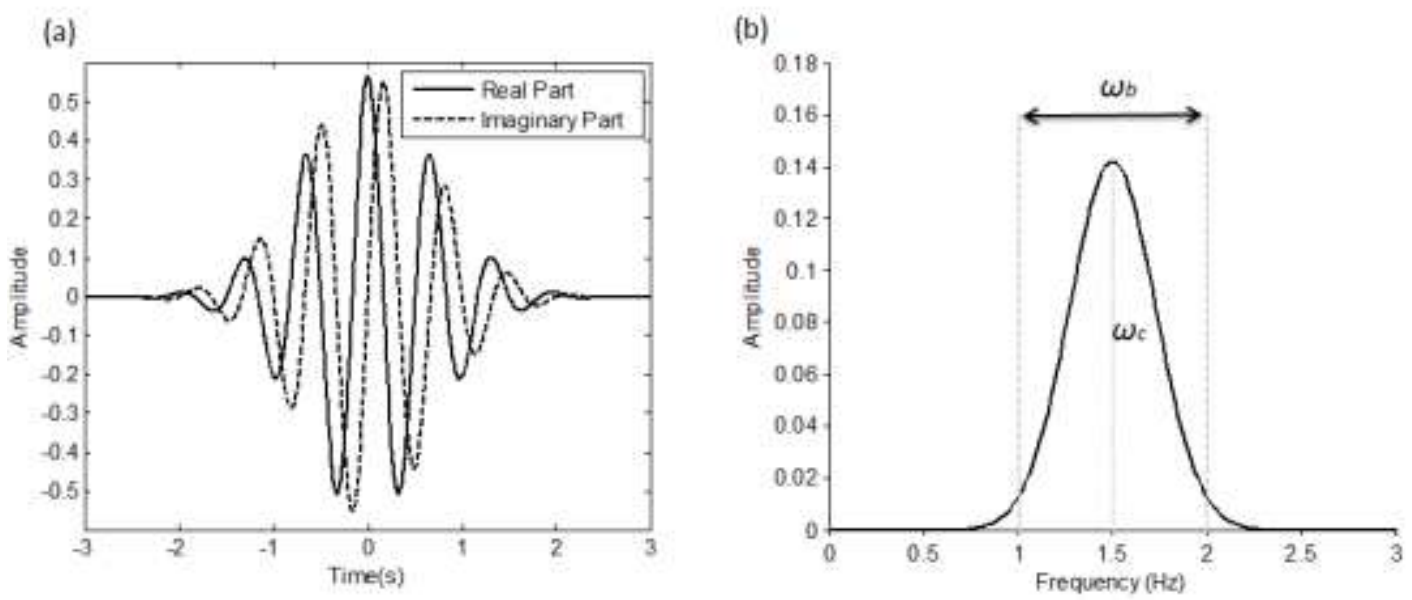

Figure 1 Complex Morlet with central frequency of $1.5 \mathrm{~Hz}$ and bandwidth of $1 \mathrm{~Hz}$, (a) time domain (b) frequency domain. 


\subsection{Acoustic Nonlinearity Parameter Obtained by WT-based Method and FFT- based Method}

Two wavelet-based schemes are introduced to obtain the acoustic nonlinearity parameter; the accuracy of the proposed schemes is demonstrated by comparison with the FFT results in this section. Figure 2 shows the schematic of the proposed schemes to extract the amplitudes of the first and second harmonic frequencies. The time-frequency spectrogram is first obtained with the selected mother wavelet, after the time-history signal is recorded, and then wavelet coefficients (amplitude) of the first and second harmonics with respect to time, $A_{1}\left(t_{i}\right)$ and $A_{2}\left(t_{i}\right)$, respectively, are extracted. Here the subscript $i$ denotes the $i$-th data point. In this study, unless otherwise indicated, the time history signals consist of 2048 discrete points. The first harmonic frequency occurs at $2.014 \mathrm{MHz}$, and the second harmonic frequency is twice the first harmonic at $4.028 \mathrm{MHz}$, as shown in Figure 2a. Two red lines in Figure 2b show the positions of first and second harmonics on the wavelet spectrum. Once the two modes are decomposed, the acoustic nonlinearity parameter can be obtained by two different schemes: (1) time-dependent:

$\beta\left(t_{i}\right)=\frac{A_{2}\left(t_{i}\right)}{A_{1}^{2}\left(t_{i}\right)}$ and (2) time-invariant: $\beta=\frac{\max \left|A_{2}\left(t_{i}\right)\right|}{\max \left|A_{1}\left(t_{i}\right)\right|^{2}}$. In the time-dependent algorithm, $\beta$

varies with each discrete point, $t_{i}$, along the time history signal. The algorithm provides a detailed variation of $\beta$ values with respect to time. However, it may include undesired wave modes and noises and, moreover, it fails when $A_{1}\left(t_{i}\right)$ is close to zero. In the time-invariant algorithm, the maximum amplitudes of $A_{1}\left(t_{i}\right)$ and $A_{2}\left(t_{i}\right)$, within a period of time, are selected for calculating a constant $\beta$ value for each time history signal. In practice, for a long period of signal, the time-dependent algorithm offers a whole spectrum of how $\beta$ varies with time due to different incidents. As for an isolated period of time of interest, the time-invariant algorithm offers a more consistent $\beta$ value for better interpretation. More detailed discussions about the two algorithms will be given in the following sections. 


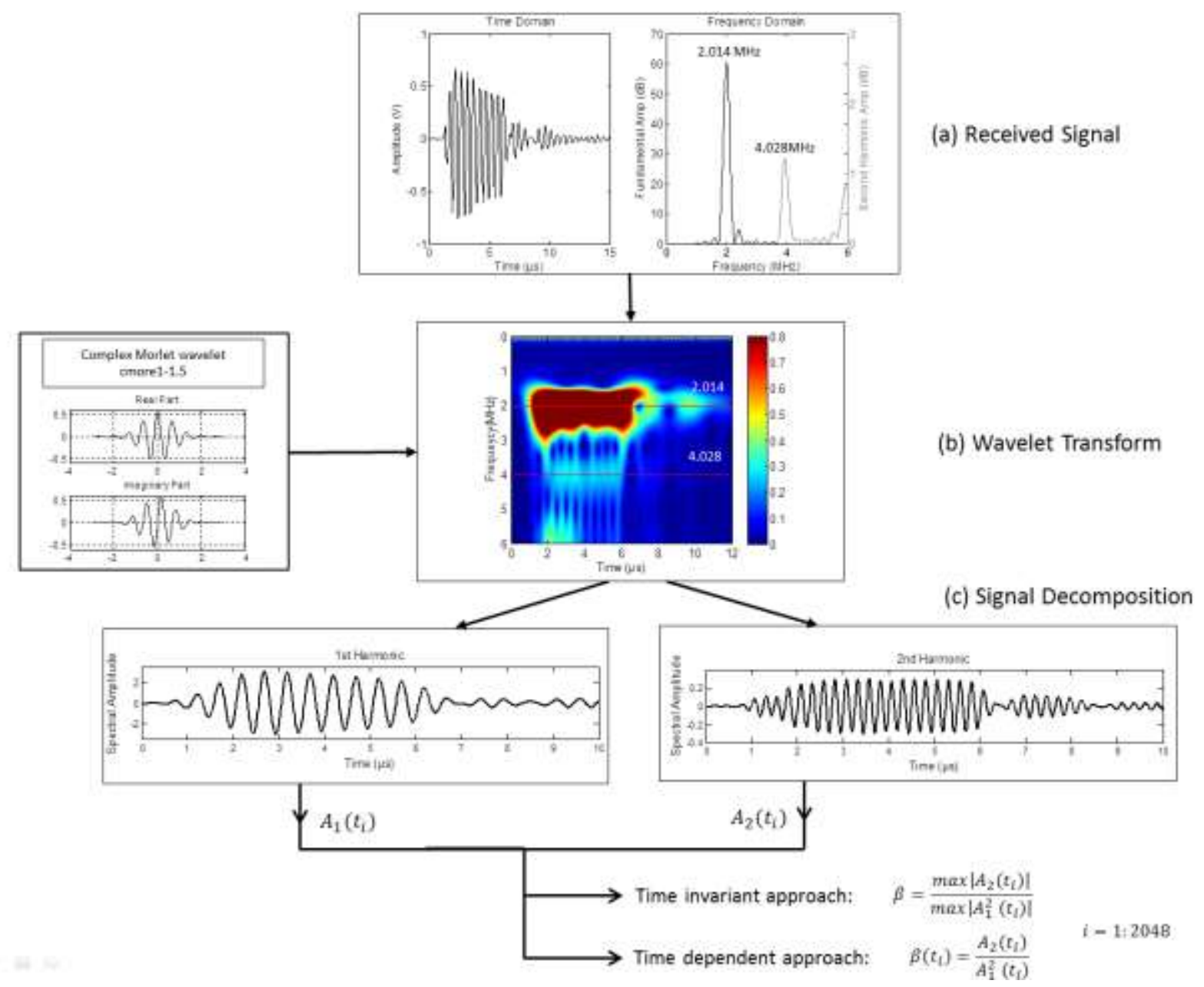

Figure 2. Schematic to obtain acoustic nonlinearity parameter $\beta$ using WT.

The acoustic nonlinearity parameter $\beta$ is an inherent material property and should not be dependent on the excitation amplitude or frequency. However, if in the signal processing the errors from the extraction of $A_{1}$ and $A_{2}$ are of the same order (see the discussion in Section 2), the error of calculated $\beta$ depends on the $A_{2}$ value. To verify the effectiveness of the waveletbased schemes for calculating the acoustic nonlinearity parameter, the analytical solution in equation (3) is employed as the input signal to completely remove measurement errors. The values of material parameters used are as follows: 
Table 1. Material parameters

\begin{tabular}{cccrcc}
\hline$A(\mathrm{~Pa})$ & $B(\mathrm{~Pa})$ & $C(\mathrm{~Pa})$ & $\boldsymbol{\rho}\left(\frac{\boldsymbol{k} \boldsymbol{g}}{\boldsymbol{m}^{3}}\right)$ & $\boldsymbol{\lambda}(\mathrm{Pa})$ & $\boldsymbol{\mu}(\mathrm{Pa})$ \\
\hline $3.51 \times 10^{11}$ & $1.444 \times 10^{11}$ & $1.028 \times 10^{11}$ & 2700 & $51.05 \times 10^{9}$ & $26.32 \times 10^{9}$ \\
\hline
\end{tabular}

The input signal $u(x, t)$ is generated according to equation (3) with various input amplitudes, $u_{0}$, and a fundamental frequency of $2 \mathrm{MHz}$. Figure 3a,b and c show $u(x, t)$ for a given $u_{0}$, its time-frequency spectrogram from WT, and its frequency domain from FFT, respectively. The variables to perform WT are $100 \mathrm{MHz}$ sampling rate and the scale of 1000, whereas the parameters to calculate FFT are $100 \mathrm{MHz}$ sampling rate, and 16384 data points. In this paper, the complex Morlet with $1.5 \mathrm{~Hz}$ central frequency $\omega_{c}$ and a bandwidth $\omega_{b}$ of $1 \mathrm{~Hz}$ is implemented. The scale $a$ in equation (13) is increased from 500 to $10 \mathrm{k}$ and the convergence in the spectrogram is reached at the scale of 5000 or greater. The pseudo frequency $F_{a}$ is $30 \mathrm{kHz}$ with a scale of 5000 and sampling frequency of $100 \mathrm{MHz}$ [52].

$$
F_{a}=\frac{\omega_{c}}{a \delta}
$$

Figure 4 compares the calculated acoustic nonlinearity parameter $\beta$ from the wavelet-based scheme (maximum amplitude) with that from the FFT. The acoustic nonlinearity parameter $\beta$ is calculated by reading $A_{1}$ and $A_{2}$ in the frequency domain (Figure 3c) when FFT is used, and it is calculated following the procedure in Figure 2 when the wavelet-based scheme is used. It can be seen in

Figure 4 that the calculated acoustic nonlinearity parameter depends on the signal amplitude in the FFT method while it is almost invariant in the WT method. It is also noted that a jump in $\beta$ value occurs in the FFT result when $A_{1}$ becomes smaller than $A_{2}$. 
(a)

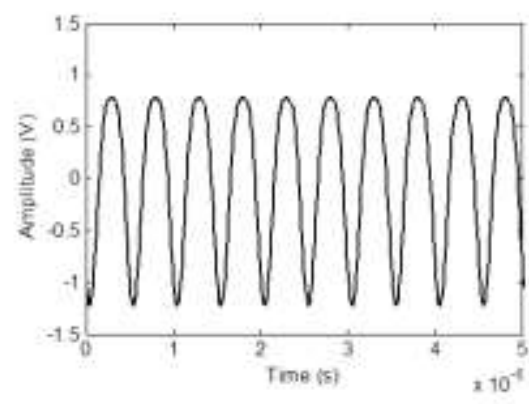

(b)

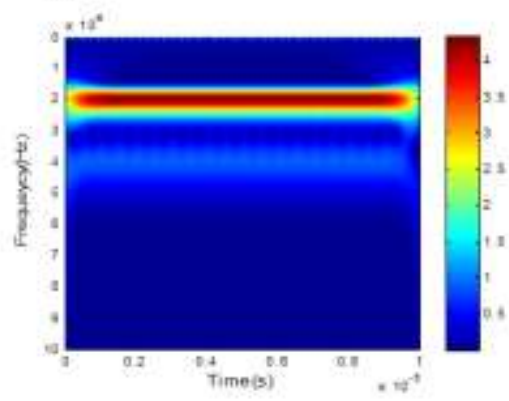

(c)

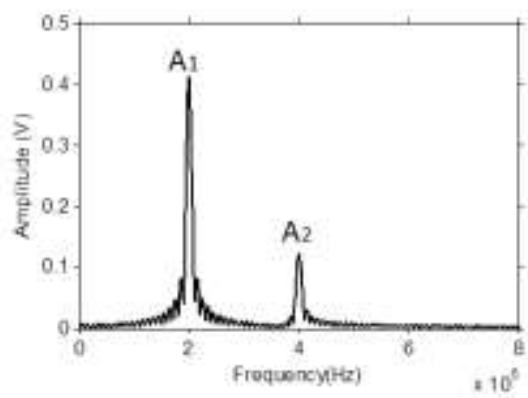

Figure 3. (a) Analytical nonlinear waveform of longitudinal wave, (b) WT, and (c) FFT.

(a)

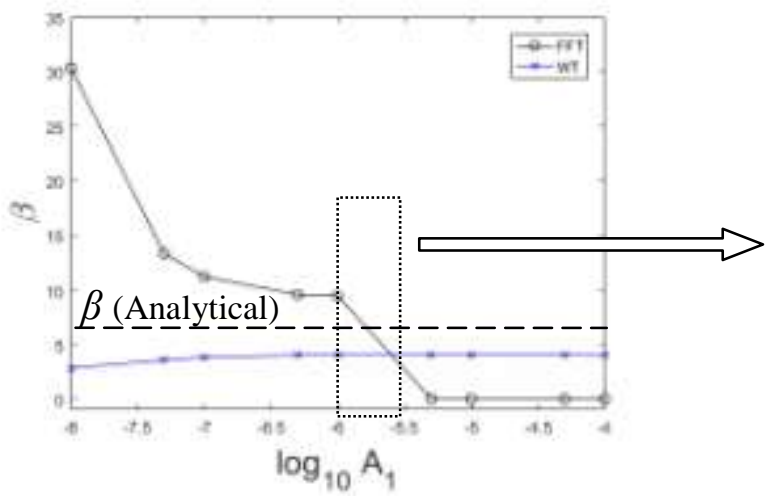

(b)

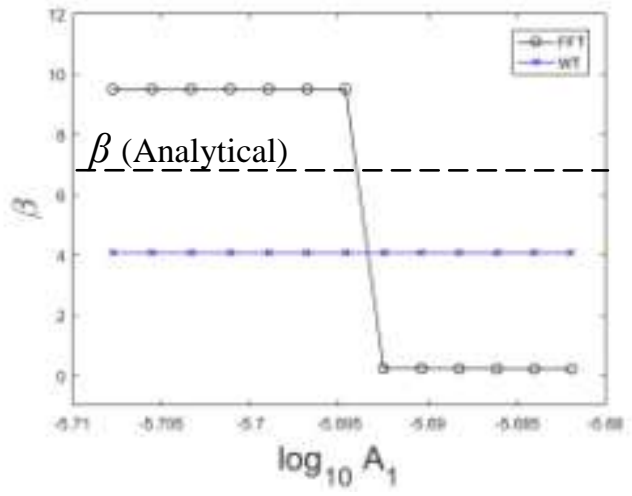

Figure 4. A comparison of the acoustic nonlinearity parameter calculated with the signal processed by FFT and WT, (a) broad range of $A_{1}\left(\sim 10^{-8}\right.$ to $\left.\sim 10^{-4}\right)$ and (b) zoomed in

$$
A_{1}\left(\sim 10^{-6}\right) \text {. }
$$

\section{Experimental Results}

In this section the experimental results on extracting the acoustic nonlinearity parameters from the samples with different levels of plastic strain are presented using different signal processing methods.

\subsection{Materials Preparation}


Aluminum 1100 was used in this investigation with a composition shown in Table 2. Nine tensile specimens were machined from a $6.3 \mathrm{~mm}(0.25$ in.) thick cold rolled plate according to ASTM standard E8 to the dimensions shown in Figure 5. All samples were stress relieved at $250^{\circ} \mathrm{C}$ for 15 minutes prior to tensile testing. An MTS tensile machine, model 1125 was used for the tensile tests using a strain rate of $2.54 \mathrm{~mm} / \mathrm{min}$. The first sample was tested to failure to obtain the stress-strain curve and to determine the yield and tensile strengths, as well as the Young's Modulus and the strain at the ultimate tensile stress (UTS), which are reported in Table 3. The remaining samples were plastically deformed between $0.5 \%$ and $4 \%$ at $0.5 \%$ strain increments to produce different uniform plastic strains through the strain gauge area to avoid non-uniform plastic deformation at the onset of necking at the ultimate tensile strength (UTS). Figure 6 shows the stress-strain curves for all the samples tested.

Table 2. Nominal chemical composition of aluminum 1100

\begin{tabular}{cccccccc}
\hline $\begin{array}{c}\text { Aluminum } \\
\mathbf{1 1 0 0}\end{array}$ & Al & $\mathbf{C u}$ & $\mathbf{M n}$ & $\mathbf{P}$ & $\mathbf{S i}+\mathbf{F e}$ & $\mathbf{Z n}$ & Others \\
\hline Weight\% & $99 \mathrm{~min}$ & $\begin{array}{c}0.05- \\
0.2\end{array}$ & 0.05 & $0-0.03$ & 0.95 & $0.1 \mathrm{max}$ & 0.15 \\
& & $\max$ & & $\max$ & & total \\
\hline
\end{tabular}

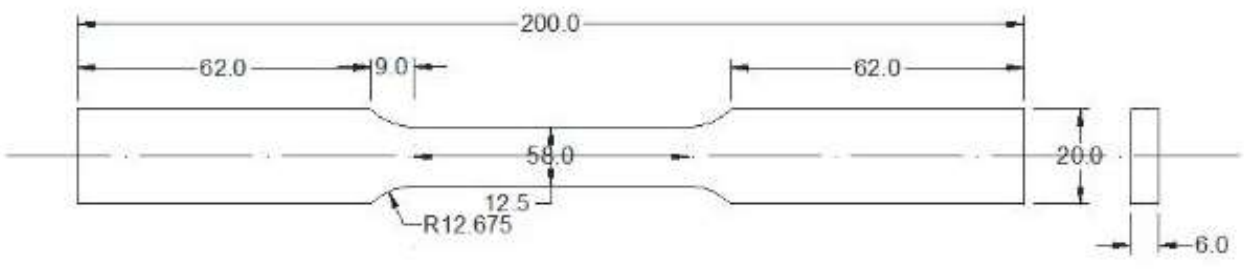

Figure 5. Tensile test sample dimensions (All dimensions are in $\mathrm{mm}$ ). 

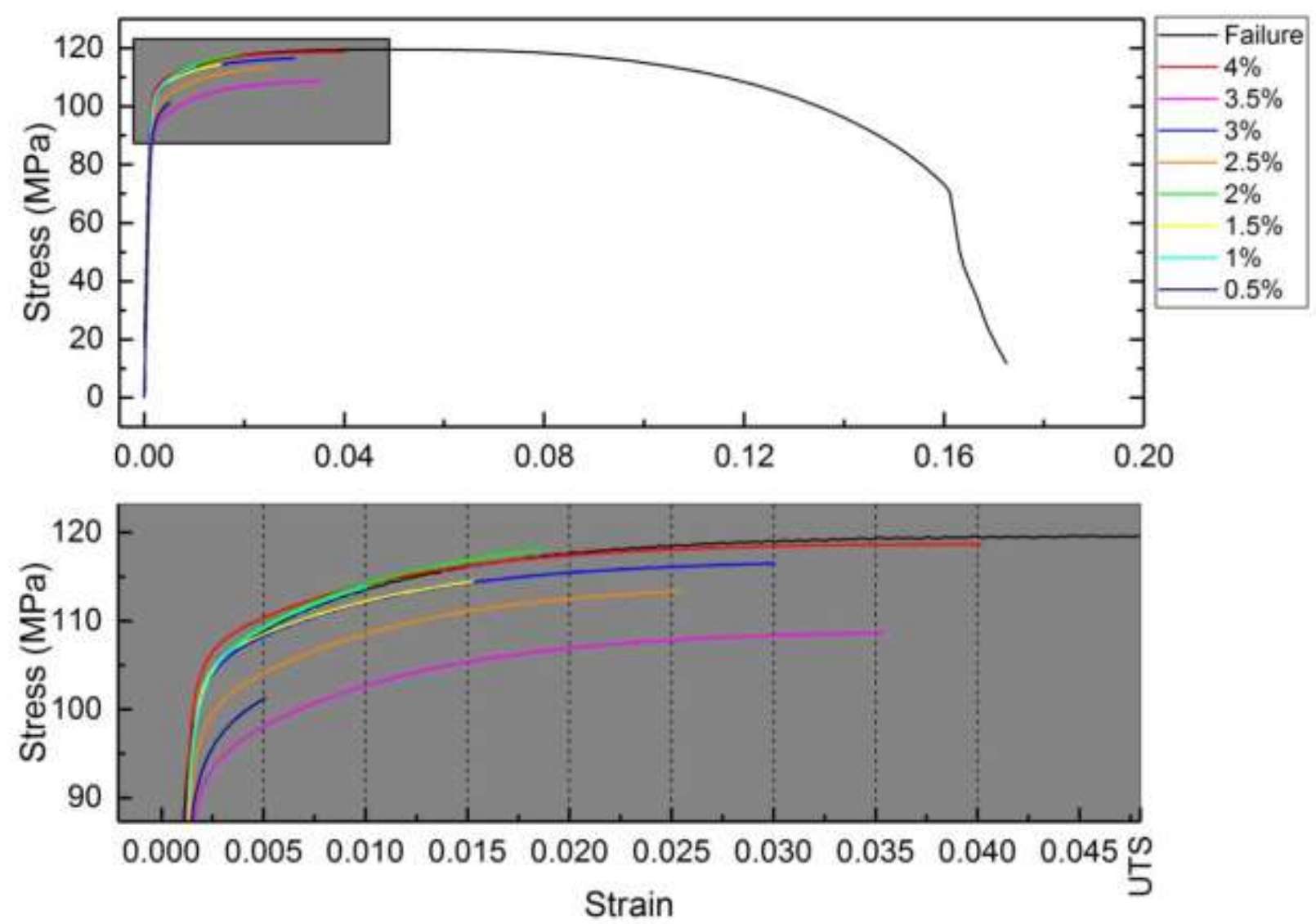

Figure 6. Stress-strain curves depicting the different strains applied to the tensile specimens.

Table 3. Experimentally determined mechanical properties of aluminum 1100

\begin{tabular}{lr}
\hline Yield Stress & $90 \mathrm{MPa}$ \\
\hline Ultimate Tensile Strength (UTS) & $120.0 \mathrm{MPa}$ \\
\hline Strain at UTS & 0.048 \\
\hline Young Modulus & $72.0 \mathrm{GPa}$
\end{tabular}

After tensile testing, the microstructures of four selected samples were examined by cross sectioning the specimens into small pieces $\left(6.0 \times 6.0 \times 5.0 \mathrm{~mm}^{3}\right)$ from the middle of the gauge length. The samples were prepared using standard metallographic procedures and etched in a 
solution of 12 parts of hydrochloric acid, six parts of Nitric acid, one part of hydrofluoric acid, and one part of distilled water. The microstructure of the selected samples was examined using light optical microscopy. Quantitative metallography was conducted using ImageJ software [53] to measure the population of intermetallics due to impurities, both in size and in volume fraction. This measurement was conducted because it has been reported in the literature [54] that in workhardenable aluminum alloys the acoustic nonlinearity parameter is a function of the second phase volume fraction. The volume fraction measurements of the second phase particles are constant for all the test specimens in our investigation (Figure 7), and this eliminates the possible effect of second phase presence on the acoustic nonlinearity parameter. Figure 8 illustrates the sequential image steps followed in the ImageJ software to calculate the volume fraction of the second phase.

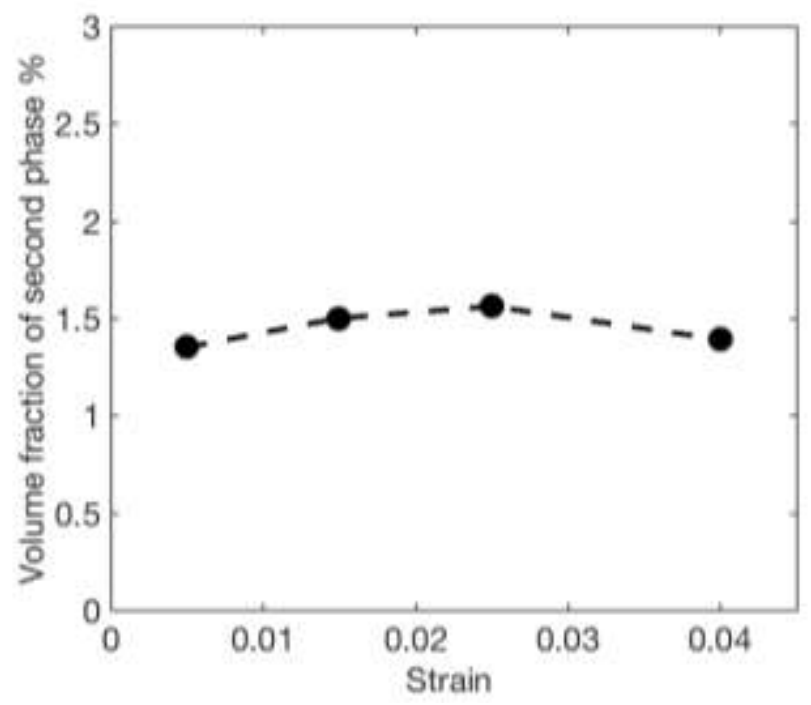

Figure 7. Second phase volume fraction measurements for the four aluminum 1100 samples. 
(a)

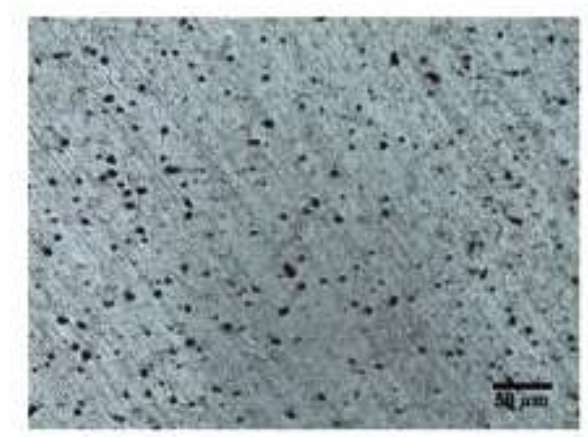

(b)

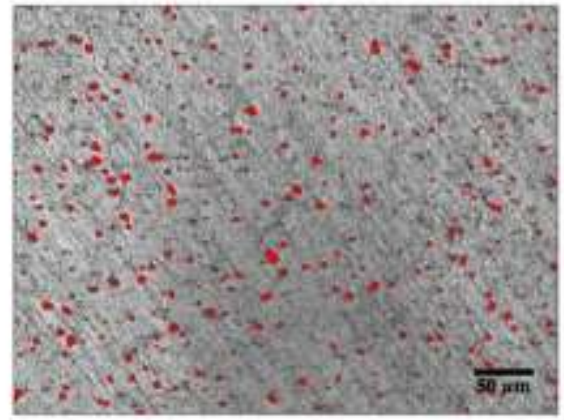

(c)

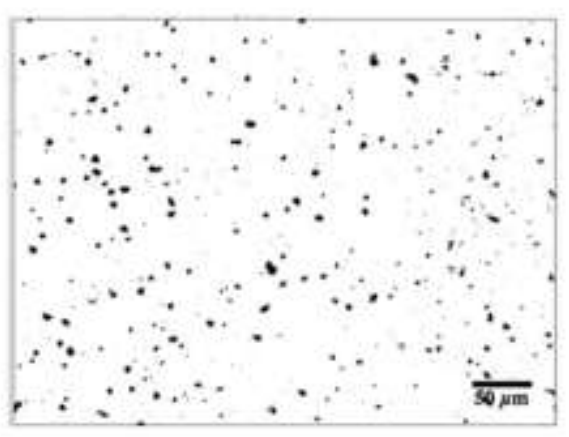

Figure 8. (a) Original micrograph of aluminum 1100, (b) and (c) imaging steps by ImageJ software to calculate volume fraction of second phase.

\subsection{Ultrasonic Testing}

There are two modes of ultrasonic testing: through-transmission and pulse echo. Separate transducers are needed for transmitting a signal to a structure and receiving the propagating waves in the structure for the through-transmission mode. The transmitting transducer is placed on one side of the structure, and the receiving transducer is placed on the opposite side of the structure. For the pulse-echo mode, the same transducer is used as both transmitter and receiver. As nonlinear ultrasonic method is based on detecting higher-harmonic signals, the throughtransmission mode should be selected for tuning the receiving transducer to the higher harmonics of the transmitting transducer (Figure 9a,b). The schematic ultrasonic testing setup in throughtransmission mode is shown in Figure 9b.

In order to provide a consistent coupling force and minimize the coupling error as discussed by Liu et al. [55], a weighted grip (24.5 N) was used to hold the transducers aligned to each other 
(Figure 9c). Light lubrication oil was used as the couplant between the transducers and the test specimens (Figure 9c). The experiments were repeated three times with recoupling the ultrasonic transducers between each measurement to check the repeatability of the results.

The transmitting and receiving transducers used in this study are piezoelectric transducers manufactured by Olympus and have effective diameter of $0.95 \mathrm{~cm}(0.375 \mathrm{inch})$ with the central frequencies of $2.25 \mathrm{MHz}$ (transmitter) and $5 \mathrm{MHz}$ (receiver), and the calibration curves shown in Figure 9d.

The major inputs to the data acquisition of nonlinear ultrasonic testing operating in throughtransmission mode are input voltage, excitation frequency, and cycles in harmonic loading. In this study, the input signal was a 10-cycle 100-volt tone burst (i.e., harmonic signal with 10 cycles as shown in Figure 9b) at $2.25 \mathrm{MHz}$, which was generated by the Pocket UT system manufactured by MISTRAS Inc. The time-history signal of the $5 \mathrm{MHz}$ receiver was recorded using the same UT system with the sampling frequency of $100 \mathrm{MHz}$ and a band-pass filter of 1 $\mathrm{MHz}-20 \mathrm{MHz}$. To improve the signal to noise ratio (SNR), twenty signals were averaged. The signal processing was performed using MATLAB software. It is important to note that the specimen thickness was smaller than the spatial length of 10-cycle tone burst signal, which caused the interference of incident and reflected waves. However, as the experimental conditions were preserved for testing each sample, the change in the acoustic nonlinearity parameter was correlated with the presence of plastic deformation. 
(a)

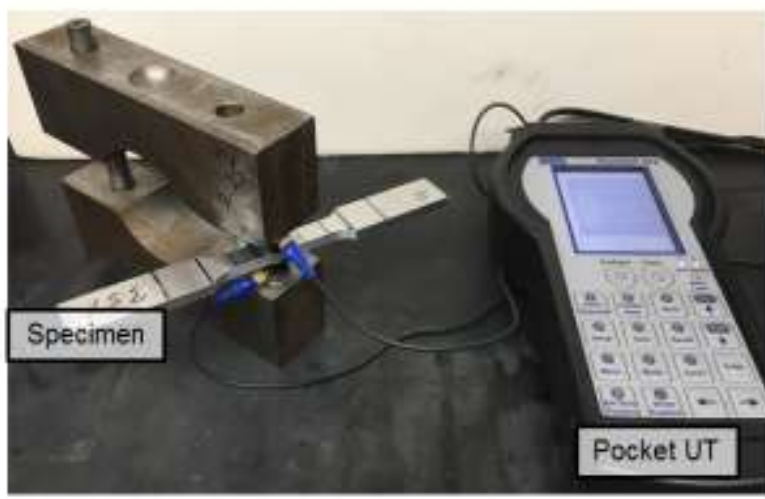

(b)

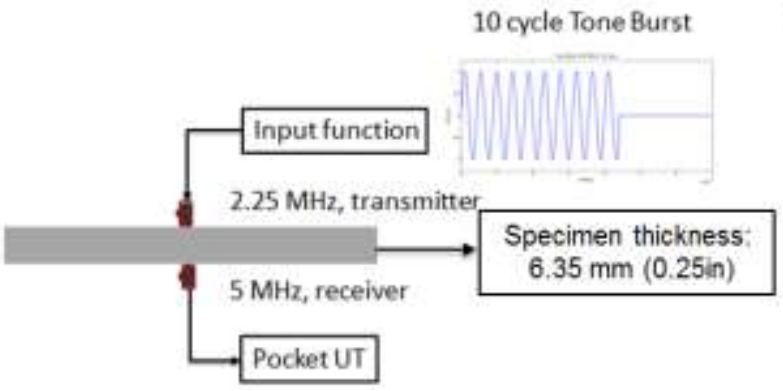

(c)

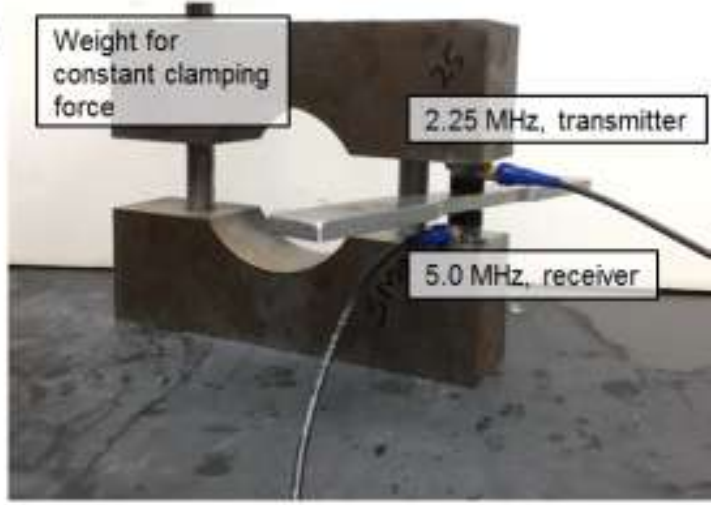

(d)

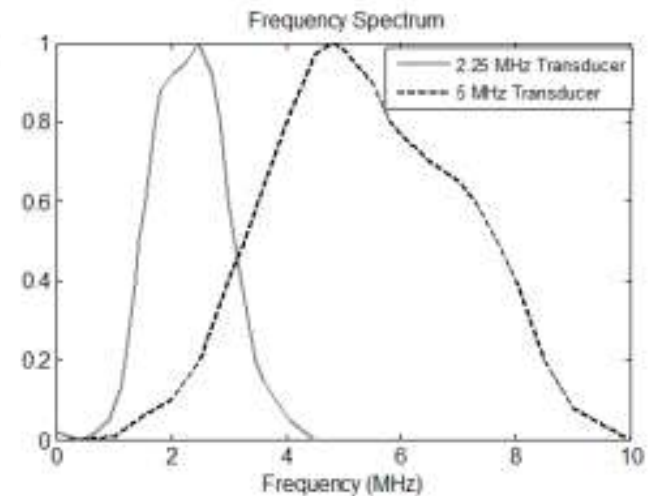

Figure 9. Ultrasonic testing of aluminum specimens, (a) experimental setup, (b) schematic diagram, (c) ultrasonic transmitter and receiver in through-transmission mode, and (d) transducer calibration curves.

\subsection{Nonlinear Ultrasonic Results using the FFT-based Method}

Once the aluminum specimens were loaded up to different strain levels and released with different permanent plastic strains, they were tested using longitudinal ultrasonic waves in a through-transmission mode to correlate the plastic deformation with the acoustic nonlinearity parameter. As discussed above, the acoustic nonlinearity parameter depends on the amplitude ratio of the first and second harmonic frequencies (equation (4)). An example of time-domain and frequency-domain signals is shown in Figure 10. The waveform is obtained from the pristine, $2 \%$ strained, and $4 \%$ strained samples and the amplitude of the second harmonic signal $(\sim 1.5)$ near $4 \mathrm{MHz}$ is significantly lower than the amplitude of the first harmonic signal ( 65) near $2 \mathrm{MHz}$. 
(a)
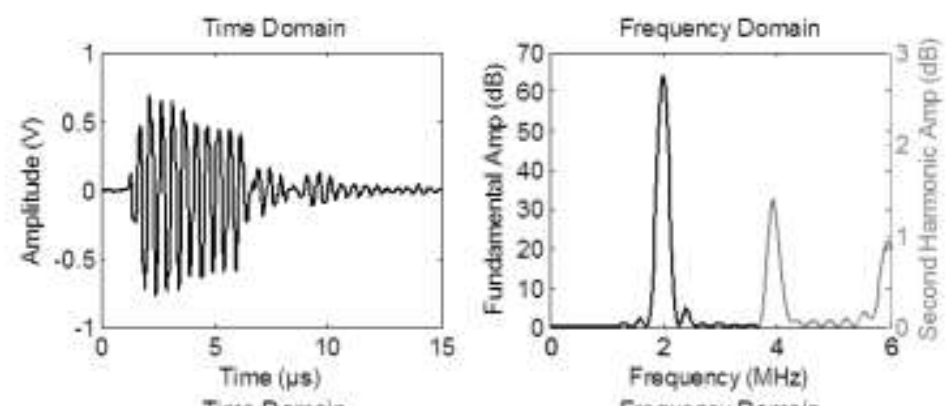

(b)
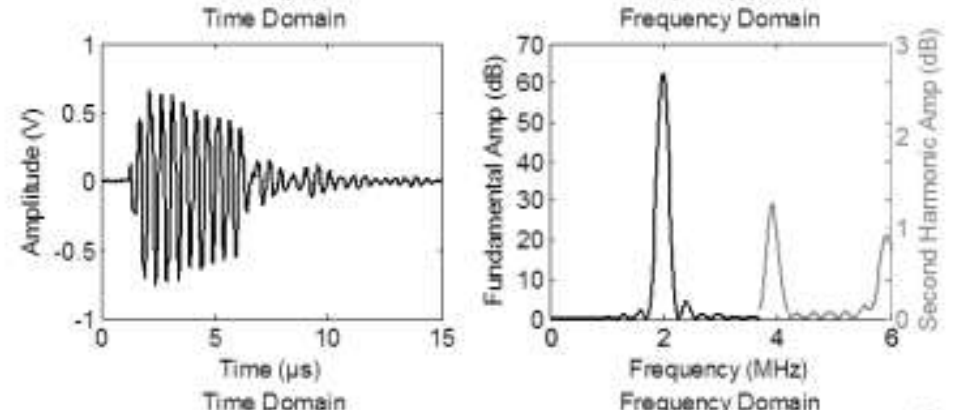

(c)
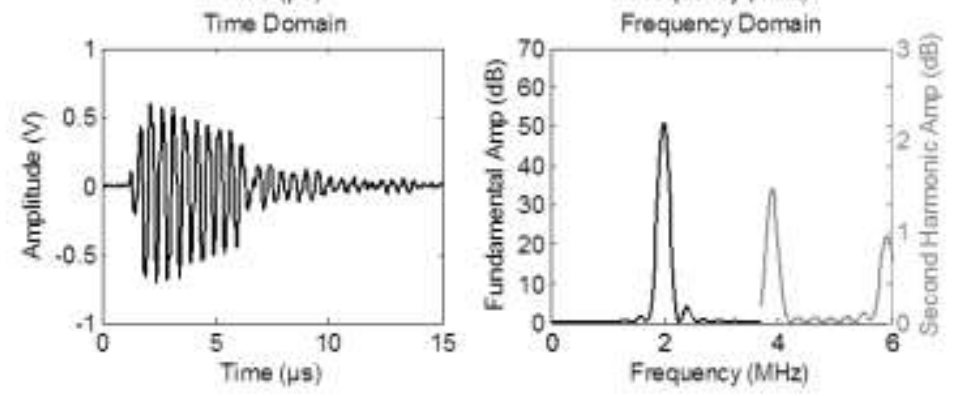

Figure 10. Typical time-domain waveforms and their frequency spectra for (a) pristine specimen,

(b) $2 \%$ strain specimen, and (c) $4 \%$ strain specimen.

It is observed that the amplitudes of harmonic frequencies highly depend on the window selected in the time domain. Figure 11 shows the normalized acoustic nonlinearity parameter calculated using the $A_{1}$ and $A_{2}$ amplitudes obtained by conducting FFT by selecting different time windows. The gray dashed lines show the window selected to compute the Fourier transform. As the ultrasonic nonlinearity due to plastic deformation is weak, the slight changes in the amplitudes of harmonic frequencies significantly affect the result. As shown in Figure 11, when the window is selected as (a) $0-15 \mu \mathrm{s}$, the acoustic nonlinearity parameter increases with the increase of plastic strain. However, when the window is changed to (b) 0-9 $\mu \mathrm{s}$, (c) 1.2-7.0 $\mu \mathrm{s}$, or 2.0-5.5 $\mu \mathrm{s}$, the correlation between the ultrasonic nonlinearity parameter and the plastic strain does not exist. Table 4 shows the values of the acoustic nonlinearity parameter obtained by different time windows. The last row in Table 4 shows the maximum percentile variation of the calculated 
nonlinearities for each specimen compared to the mean value of the calculated nonlinearities of that specimen. For the same data set, the acoustic nonlinearity parameter can change more than $100 \%$ by varying the time window. Therefore, identifying the acoustic nonlinearity parameter by FFT introduces significant error in the measurement.
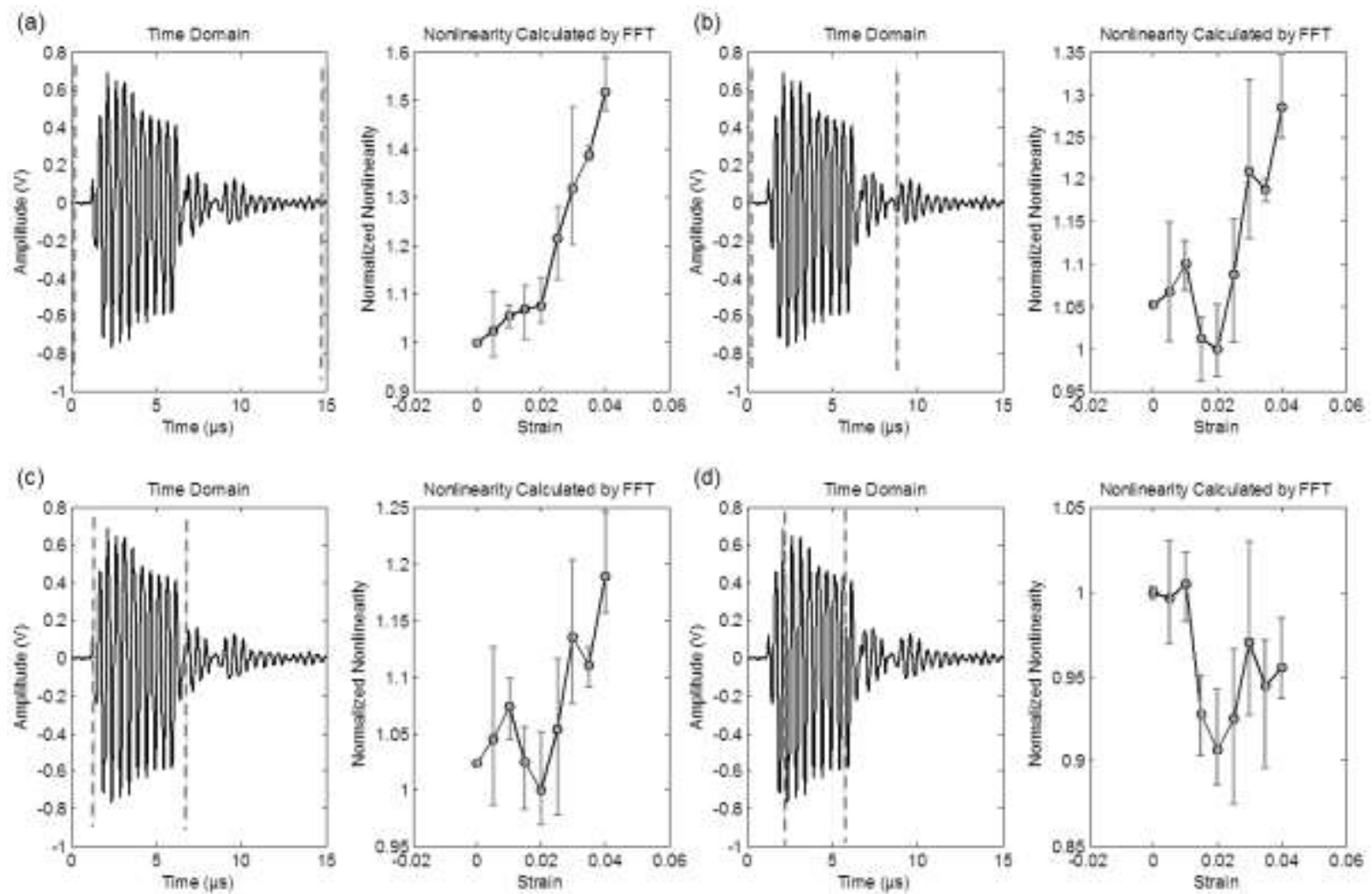

Figure 11. The variations in the acoustic nonlinearity parameter corresponding to time-domain windows calculated by FFT, (a) $0-15 \mu \mathrm{s}$, (b) $0-9 \mu \mathrm{s}$, (c) $1.2-7.0 \mu \mathrm{s}$ and (d) $2.0-5.5 \mu \mathrm{s}$.

Table 4. Acoustic nonlinearity parameter obtained by FFT

\begin{tabular}{ccccccc}
\hline \multirow{2}{*}{$\begin{array}{c}\text { Start } \\
\text { Time }(\boldsymbol{\mu s})\end{array}$} & $\begin{array}{c}\text { Finish } \\
\text { Time }(\boldsymbol{\mu s})\end{array}$ & \multicolumn{5}{c}{ Acoustic Nonlinearity Parameter } \\
\cline { 3 - 7 } & Pristine & 0.01 & 0.02 & 0.03 & 0.04 \\
\hline 0 & 15 & 0.272 & 0.287 & 0.293 & 0.359 & 0.414 \\
\hline 0 & 9 & 0.147 & 0.154 & 0.140 & 0.169 & 0.180 \\
\hline 1 & 8 & 0.106 & 0.111 & 0.104 & 0.124 & 0.132 \\
\hline 1.5 & 7.5 & 0.092 & 0.095 & 0.088 & 0.102 & 0.111 \\
\hline
\end{tabular}




\begin{tabular}{ccccccc}
\hline 2 & 7 & 0.091 & 0.091 & 0.084 & 0.091 & 0.090 \\
\hline 2.5 & 6.5 & 0.088 & 0.089 & 0.083 & 0.091 & 0.092 \\
\hline 3 & 6 & 0.114 & 0.116 & 0.104 & 0.115 & 0.117 \\
\hline & \%change & 109.193 & 113.293 & 128.937 & 139.126 & 155.042 \\
\hline
\end{tabular}

\subsection{Nonlinear Ultrasonic Results using the WT-based Method}

Figure 12 shows the time histories of wavelet coefficients for the first and second harmonic frequencies for four different plastic strains. In the first approach, the peak amplitudes are identified without preserving the time information, and the acoustic nonlinearity parameters of samples with the induced plastic strains are calculated. Figure 13 shows the stress-strain curve for the aluminum 1100 specimen together with the normalized acoustic nonlinearity parameter of each sample. As the thickness changes in different plastic strain levels, the thickness of each sample was measured and taken into account to calculate the acoustic nonlinearity parameter. These coefficients are plotted as a function of strain as shown in Figure 13. It is observed that the acoustic nonlinearity parameters exhibit stationary behavior up to $1 \%$ strain. A rapid increase in the acoustic nonlinearity parameter occurs between $1 \%$ strain and $3.5 \%$ strain, but then saturation in the acoustic nonlinearity parameter ensues near 3.5\% strain, which is close to the necking point. The ultrasonic measurement was repeated three times for each sample. Each point in Figure 13 represents the mean value, and the corresponding error bar represents the range of variation in the three measurements. To show the trend of the experimental results, the spline curve is fitted to the experimental data. The interpolation method in the spline is piecewisepolynomial interpolation that reduces the interpolation error compared to polynomial interpolation [56]. 
(a)
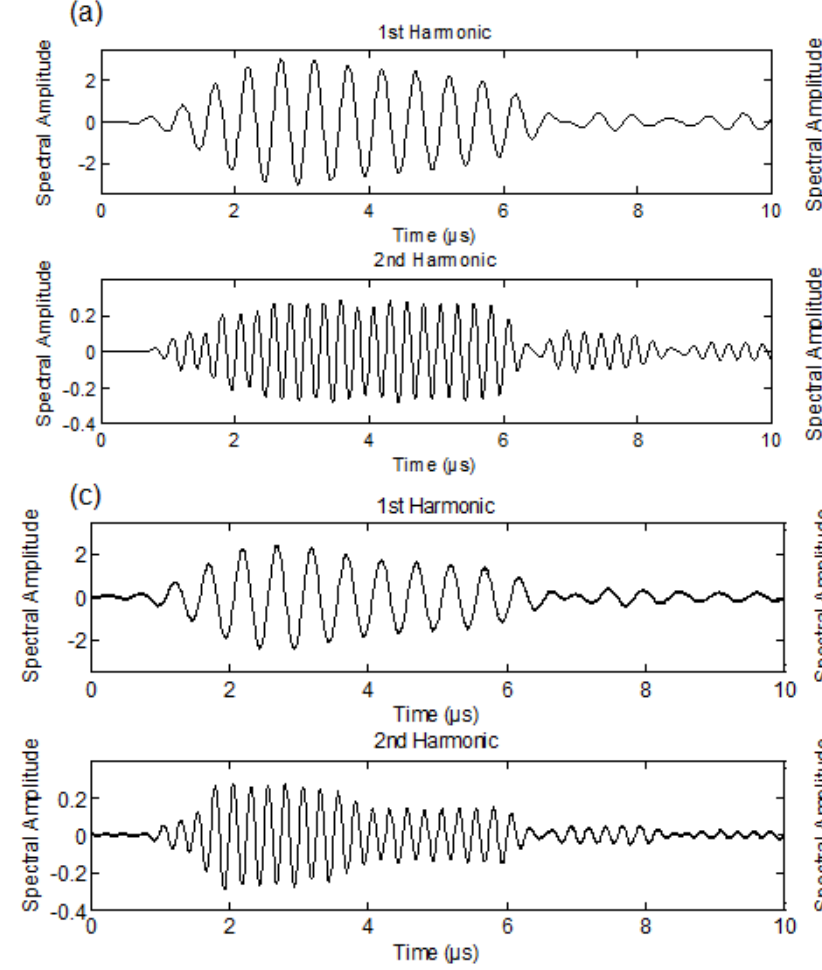

(b)
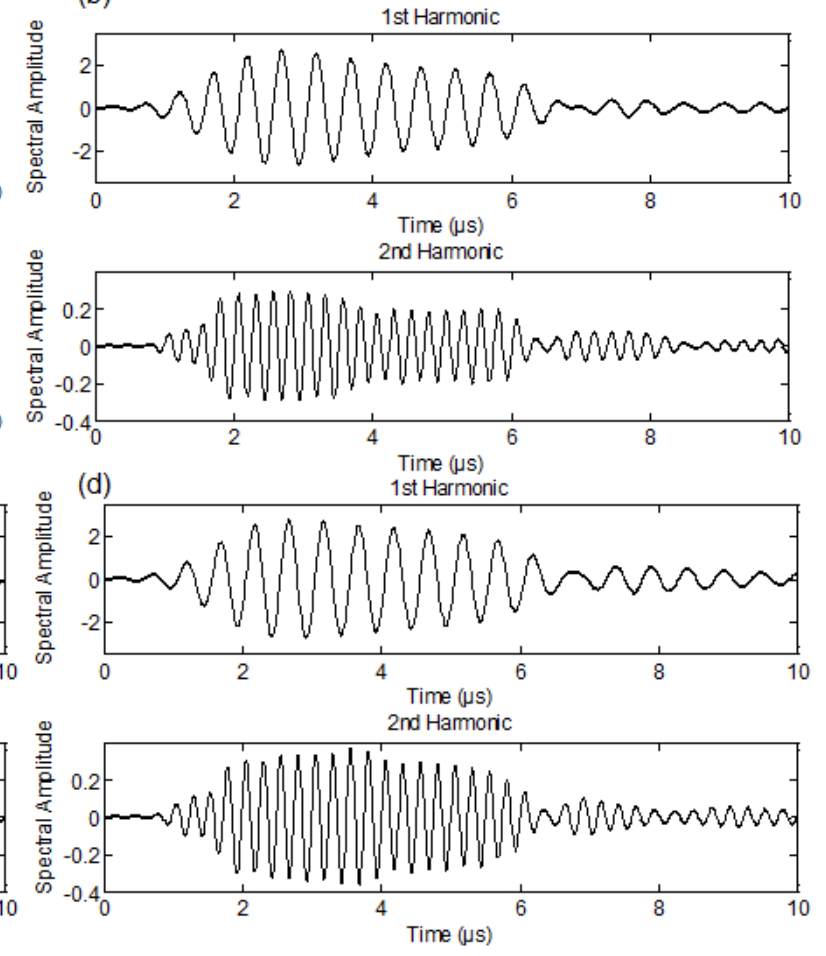

Figure 12. Fundamental and second harmonic waveforms extracted from the spectrograms corresponding to samples, (a) pristine, (b) 2\% strained, (c) 3\% strained, and (d) 4\% strained. 


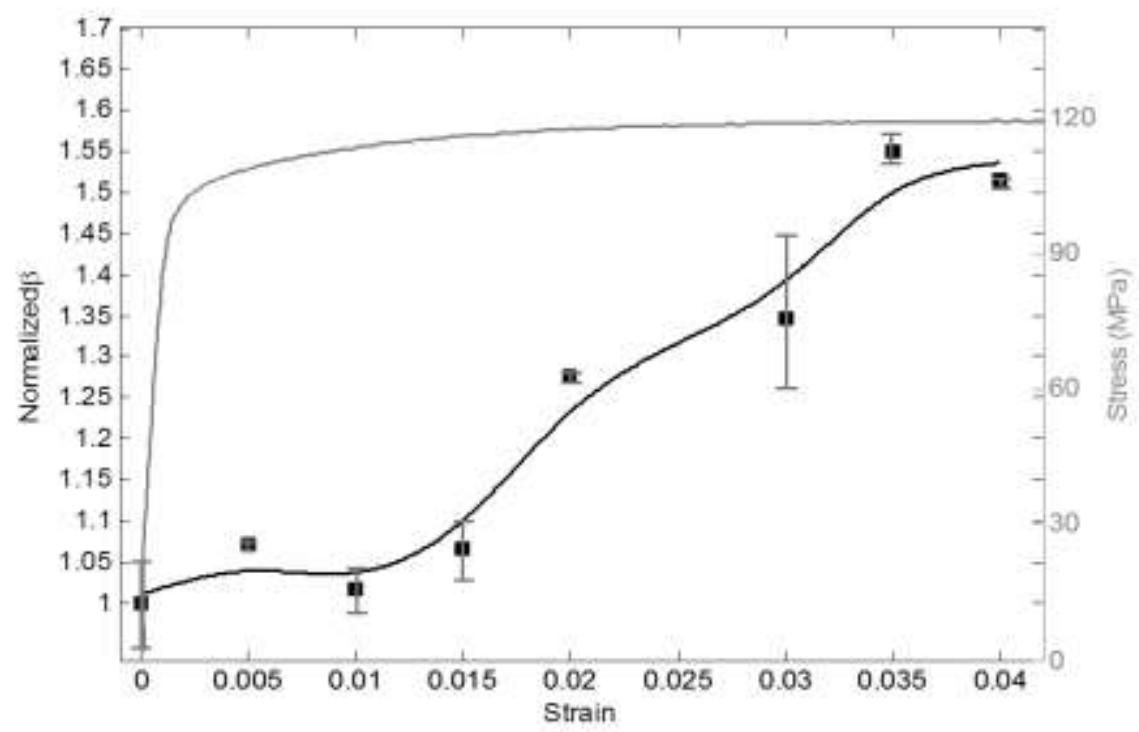

Figure 13. Stress-strain and normalized nonlinearity-strain curves on two-scale plot.

The second approach to identify the acoustic nonlinearity parameter using the time histories of wavelet coefficients for the first and second harmonics is based on obtaining the wave envelopes at each frequency, and applying equation (4) to the entire time-history data as shown in Figure 14 for the pristine specimen. While the peak amplitudes of each frequency component do not occur at the same time, the acoustic nonlinearity parameter shows a constant regime within the time interval $2.0-5.5 \mu \mathrm{s}$.

The same approach was repeated for the rest of the specimens that underwent different levels of plastic strain as shown in Figure 15. In general, the time-dependent acoustic nonlinearity parameter increases with the increase of plastic strain while some fluctuations are observed in the data set.

All the acoustic nonlinearity parameters were averaged in the time interval of 2.6 -3.6 $\mu$ s and plotted as a function of the amount of strain as presented in Figure 16, along with the stressstrain curve for a pristine aluminum 1100 sample. The results of both wavelet-based algorithms, time- invariant and time- dependent, link the acoustic nonlinearity parameter and plastic strain in 
the material. The acoustic nonlinearity parameter increases by $55 \%$ in the time-invariant algorithm, and $45 \%$ in the time-dependent algorithm.

(a)

(b)

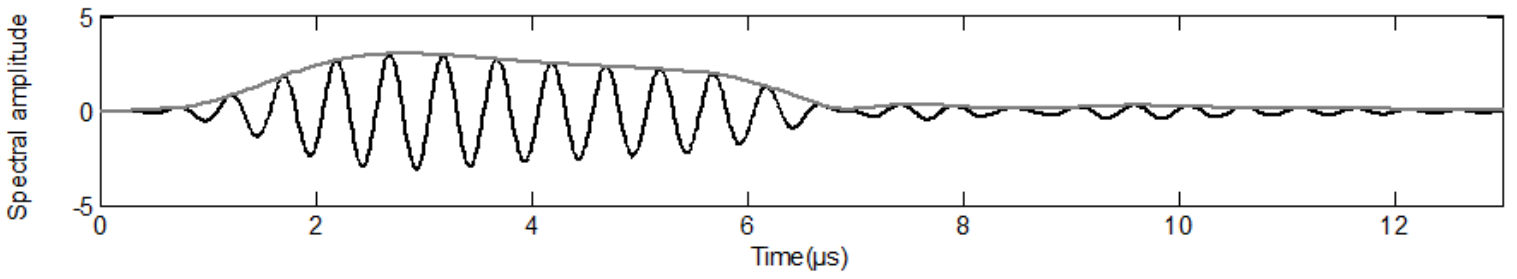

(c)
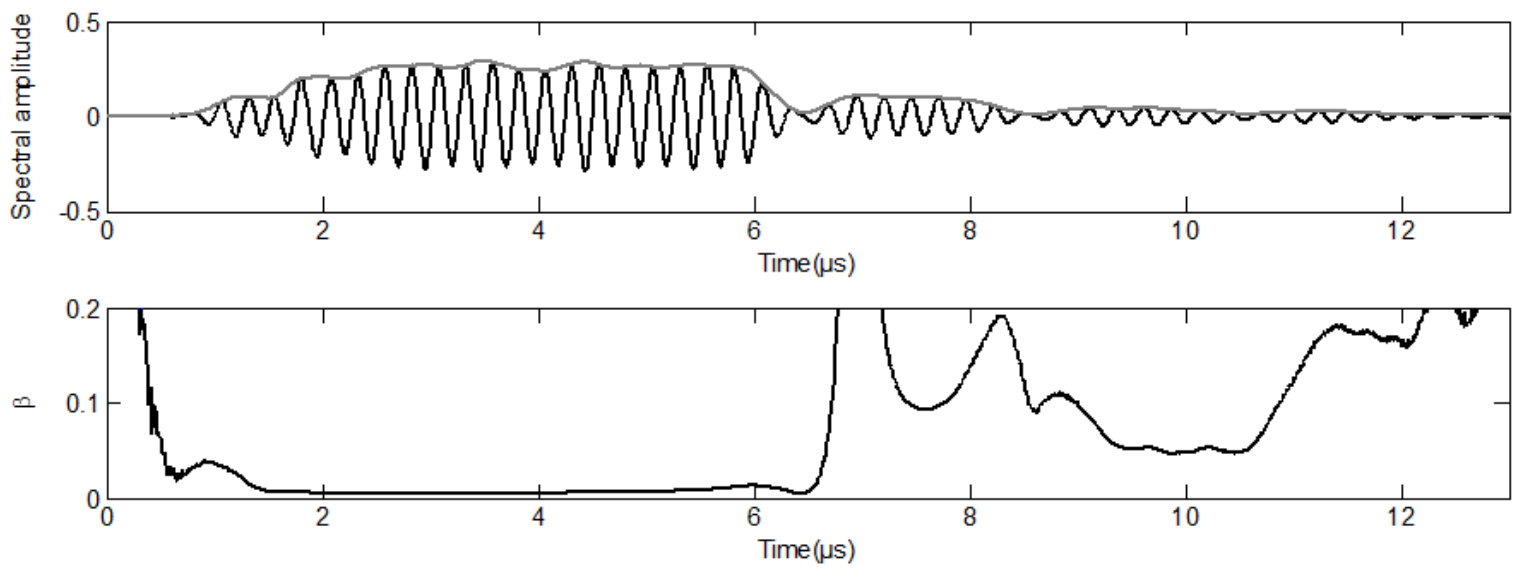

Figure 14. (a) Time-history waveform of the first harmonic, (b) time-history waveform of the second harmonic, and (c) change in acoustic nonlinearity parameter with time (for pristine specimen). 


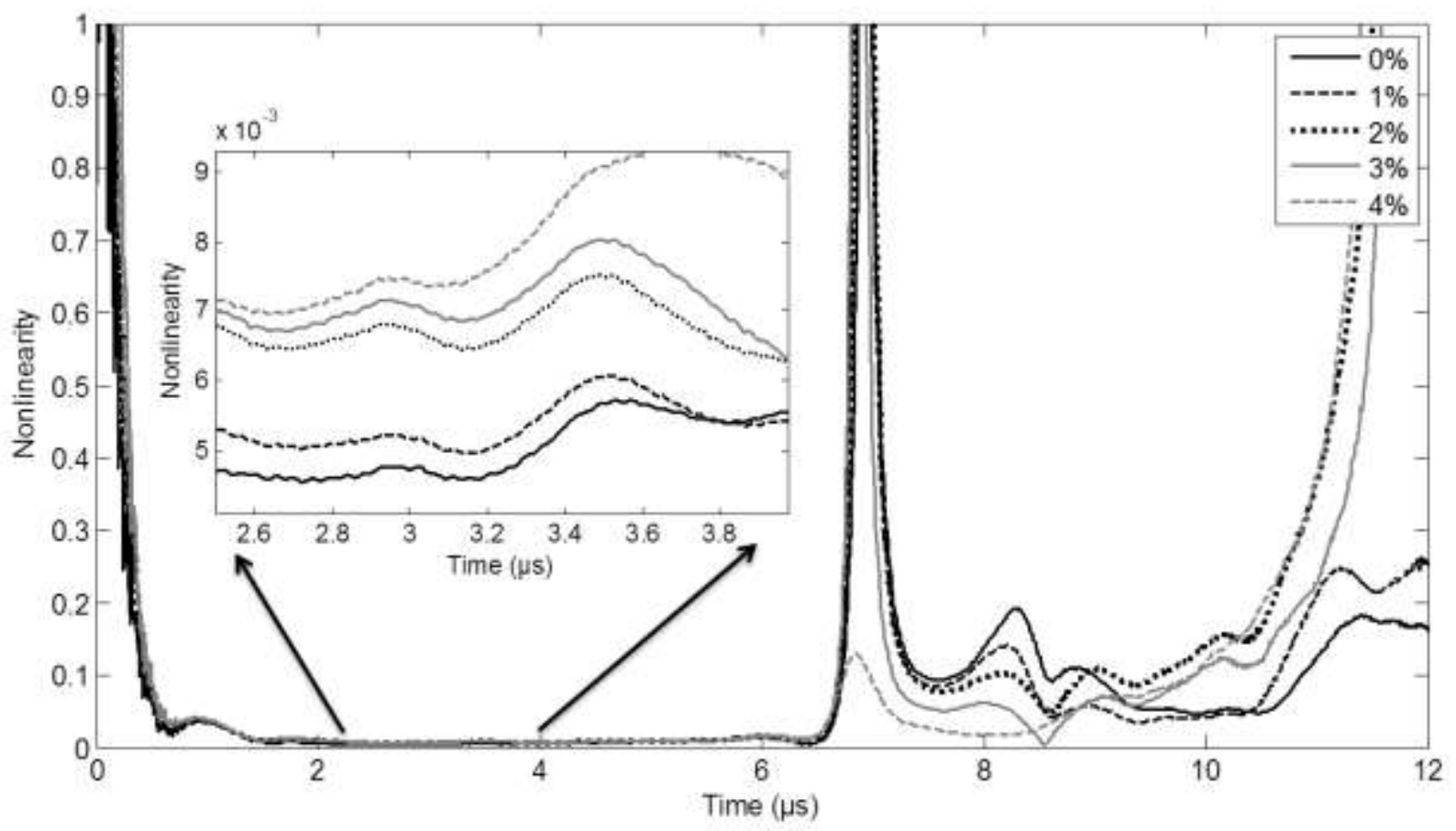

Figure 15. Change in acoustic nonlinearity parameter within time for different strain levels. 


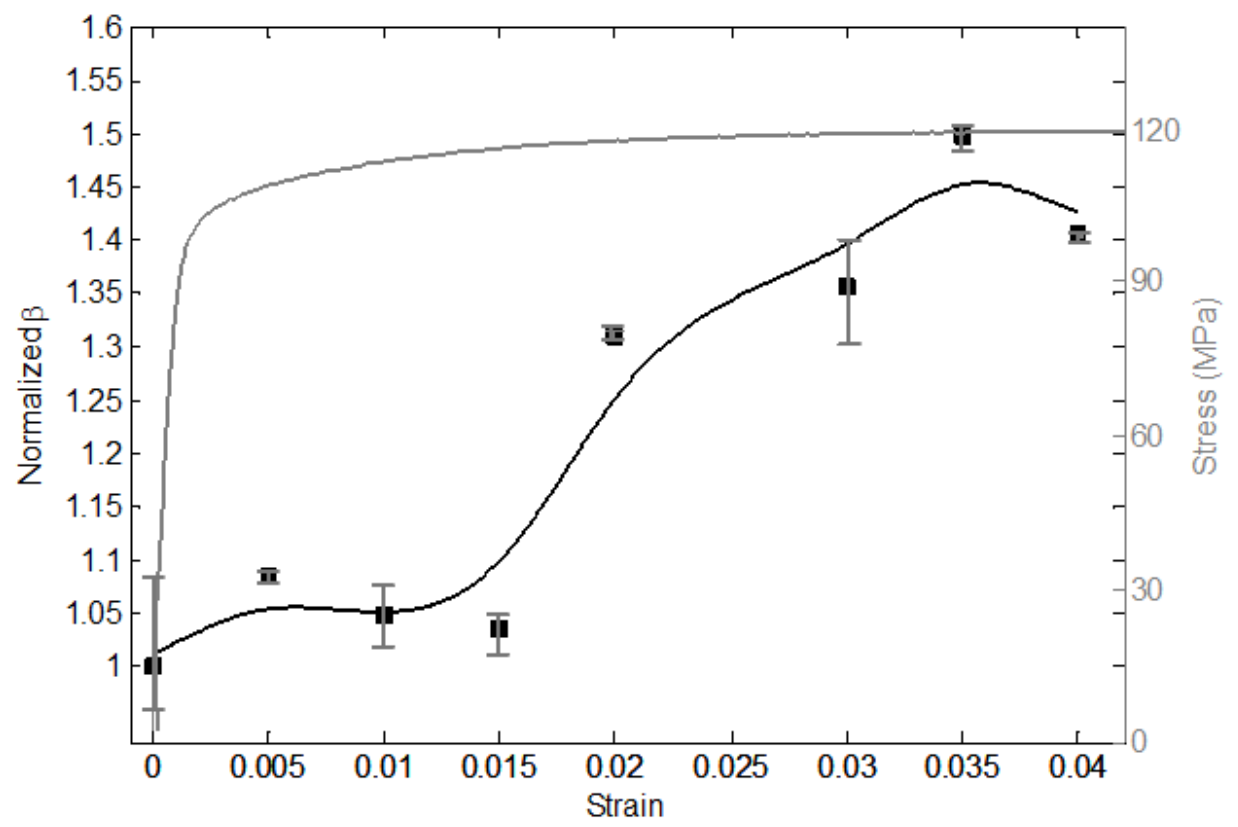

Figure 16. Acoustic nonlinearity parameter obtained by preserving time information in WT.

\section{Conclusions}

The wavelet-based algorithms are introduced to obtain the acoustic nonlinearity parameter $\beta$. The comparison between the signal processing methods based on the FFT and the WT using an analytical solution as the input signal shows that the calculated $\beta$ from the WT method agrees well with the analytical solution regardless the input amplitude while the result from the FFT exhibits strong dependence on the amplitude. The proposed wavelet-based schemes are employed to investigate the change of acoustic nonlinearity parameter $\beta$ caused by plastic deformation, for the purpose of validation. The accuracy of harmonic decomposition of nonlinear wave signal due to plastic deformation is improved by applying the proposed wavelet-based algorithms. Consequently, a good correlation between the increase of the acoustic nonlinearity parameter $\beta$ and the increase in plastic deformation is obtained in the nonlinear UT experiments. The developed signal processing algorithm can enhance the minimum detectable microstructural change in materials using nonlinear ultrasonics in addition to plastic deformation studied in this 
paper such as porosity, microstructural composition (such as ferrite content in steel), fatigue damage, creep damage, and weld defects.

\section{Acknowledgement}

This research is based upon work supported by the National Science Foundation (NSF) under award number CMMI 1463501 entitled “Assessing Microstructural Damage Using Nonlinear Ultrasonics and Multiscale Numerical Modeling". Any opinions, findings and conclusions or recommendations expressed in this paper are those of the authors and do not necessarily reflect the views of the NSF. We would like to acknowledge Dr. Daniel P. Bailey of the UIC College of Engineering for his assistance in editing this article.

\section{Appendix}

Following the equations (1) and (2), the strain energy density function for isotropic solids converts to:

$$
W=\frac{\lambda}{2}(t r \mathbf{E})^{2}+\mu t r \mathbf{E}^{2}+\frac{A}{3} \operatorname{tr} \mathbf{E}^{3}+B t r \mathbf{E} t r \mathbf{E}^{2}+\frac{C}{2}(t r \mathbf{E})^{3}+\ldots
$$

where $\lambda$ and $\mu$ are Lamé parameters and $A, B$ and $C$ are the Landau-Lifshitz representation of third-order elastic constants (TOE) [57]. If only purely longitudinal motion is considered, equation of motion is reduced to the following expression:

$$
\frac{\partial^{2} u}{\partial t^{2}}=c_{l}^{2} \frac{\partial^{2} u}{\partial x^{2}} g\left(\frac{\partial u}{\partial x}\right)
$$

where $c_{l}$ is the longitudinal wave speed and is related to material properties by:

$$
c_{l}=\sqrt{\frac{\lambda+2 \mu}{\rho_{0}}}
$$

and

$$
g(\xi)=1+\left(3+\frac{C_{111}}{\rho_{0} c_{l}^{2}}\right) \xi+\left(3+\frac{3 C_{111}+C_{1111}}{\rho_{0} c_{l}^{2}}\right) \frac{\xi^{2}}{2 !}+\ldots
$$


where $\rho_{0}$ is the density in the unreformed state and $c_{111}$ and $c_{1111}$ are the third order and fourth order elastic constants, respectively. It has been shown in references that equation (14) can be solved along its characteristics and it can be demonstrated that the wave speed in the actual space is changed due to nonlinearity by the coefficient $\beta^{\prime}$ :

$$
c+v=c_{l}+\beta^{\prime} v
$$

where $c$ is the actual wave speed and $v$ is the particle velocity. $\beta^{\prime}$ in turn, has the following relationship with TOE:

$$
\beta^{\prime}=-\left(\frac{3}{2}+\frac{C_{111}}{2 \rho_{0} c_{l}^{2}}\right)
$$

where $C_{111}=2 A+6 B+2 C$. For a given material, $\beta^{\prime}$ is calculated using equation (18) and inserted in equation (3), and then recalculated using the amplitude ratio (equation (4)) by employing the WT or the FFT.

\section{References}

[1] B. Chassignole, R. El Guerjouma, M.A. Ploix, T. Fouquet, Ultrasonic and structural characterization of anisotropic austenitic stainless steel welds: Towards a higher reliability in ultrasonic non-destructive testing, NDT E Int. 43 (2010) 273-282.

doi:10.1016/j.ndteint.2009.12.005.

[2] S. Hirsekorn, P.W. Van andel, U. Netzelmann, Ultrasonic Methods To Detect and Evaluate Damage in Steel, Nondestruct. Test. Eval. 15 (1998) 373-393. doi:10.1080/10589750008952880.

[3] A. Ebrahimkhanlou, B. Dubuc, S. Salamone, Damage localization in metallic plate structures using edge-reflected lamb waves, Smart Mater. Struct. 25 (2016) 85035.

[4] S.P. Sagar, S. Das, N. Parida, D.K. Bhattacharya, Non-linear ultrasonic technique to 
assess fatigue damage in structural steel, Scr. Mater. 55 (2006) 199-202.

doi:10.1016/j.scriptamat.2006.03.037.

[5] P.B. Nagy, Fatigue damage assessment by nonlinear ultrasonic materials characterization, Ultrasonics. 36 (1998) 375-381. doi:10.1016/S0041-624X(97)00040-1.

[6] J.H. Cantrell, W.T. Yost, Nonlinear Ultrasonic Characterization of Fatigue Microstructures, Int. J. Fatigue. 23 (2001) 487-490. doi:10.1016/S0142-1123(01)00162-1.

[7] A. Kumar, C.J. Torbet, J.W. Jones, T.M. Pollock, Nonlinear ultrasonics for in situ damage detection during high frequency fatigue, J. Appl. Phys. 106 (2009). doi:10.1063/1.3169520.

[8] E.H. Dowell, G.F. Gorman, D.A. Smith, Acoustoelasticity: General theory, acoustic natural modes and forced response to sinusoidal excitation, including comparisons with experiment, J. Sound Vib. 52 (1977) 519-542. doi:10.1016/0022-460X(77)90368-6.

[9] Z. Abiza, M. Destrade, R.W. Ogden, Large acoustoelastic effect, Wave Motion. 49 (2012) 364-374. doi:10.1016/j.wavemoti.2011.12.002.

[10] Z. Abbasi, D. Ozevin, Acoustoelastic Coefficients in Thick Steel Plates under Normal and Shear Stresses, Exp. Mech. 56 (2016) 1583-1591. doi:10.1007/s11340-016-0186-6.

[11] A.A. Buenos, P. Pereira Jr., P. Roberto, M. Auteliano, Influence of Grain Size on the Propagation of L CR Waves in Low Carbon Steel, J. Nondestruct. Eval. 33 (2014) 562 570. doi:10.1007/s10921-014-0252-x.

[12] A. Gedroits, V. Krasilnikov, Finite-amplitude elastic waves in solids and deviations from Hooke's law, Sov. Phys. JETP. 16 (1963) 1122-1126.

[13] M.A. Breazeale, J. Ford, Ultrasonic studies of the nonlinear behavior of solids, J. Appl. Phys. 36 (1965) 3486-3490. doi:10.1063/1.1703023.

[14] K. Jhang, Nonlinear Ultrasonic Techniques for Non- destructive Assessment of Micro Damage in Material : A Review, Int. J. Precis. Eng. Manuf. 10 (2009) 123-135.

[15] K.H. Matlack, H.A. Bradley, S. Thiele, J. Kim, J.J. Wall, H. Joon, J. Qu, L.J. Jacobs, Nonlinear ultrasonic characterization of precipitation in 17-4PH stainless steel, NDT E Int. 71 (2015) 8-15. doi:10.1016/j.ndteint.2014.11.001. 
[16] M.A. Breazeale, D.O. Thompson, Finite-amplitude ultrasonic waves in aluminum, Appl. Phys. Lett. 3 (1963) 77-78. doi:10.1063/1.1753876.

[17] J. Herrmann, J.Y. Kim, L.J. Jacobs, J. Qu, J.W. Littles, M.F. Savage, Assessment of material damage in a nickel-base superalloy using nonlinear Rayleigh surface waves, J. Appl. Phys. 99 (2006). doi:10.1063/1.2204807.

[18] H.J. Lim, B. Song, B. Park, H. Sohn, Noncontact fatigue crack visualization using nonlinear ultrasonic modulation, NDT E Int. 73 (2015) 8-14.

[19] C. Pruell, J. Kim, J. Qu, L.J. Jacobs, Evaluation of fatigue damage using nonlinear guided waves, Smart Mater. Struct. 18 (2009). doi:10.1088/0964-1726/18/3/035003.

[20] S. Baby, B.N. Kowmudi, C.M. Omprakash, D.V. V Satyanarayana, Creep damage assessment in titanium alloy using a nonlinear ultrasonic technique, Scr. Mater. 59 (2008) 818-821. doi:10.1016/j.scriptamat.2008.06.028.

[21] K. Balasubramaniam, J.S. Valluri, R. V Prakash, Creep damage characterization using a low amplitude nonlinear ultrasonic technique, Mater. Charact. 62 (2010) 275-286. doi:10.1016/j.matchar.2010.11.007.

[22] Y. Xiang, W. Zhu, C.-J. Liu, F.-Z. Xuan, Y.-N. Wang, W.-C. Kuang, Creep degradation characterization of titanium alloy using nonlinear ultrasonic technique, NDT E Int. 72 (2015) 41-49. doi:10.1016/j.ndteint.2015.02.001.

[23] A. Ruiz, N. Ortiz, A. Medina, J. Kim, L.J. Jacobs, Application of ultrasonic methods for early detection of thermal damage in 2205 duplex stainless steel, NDT E Int. 54 (2013) 19-26. doi:10.1016/j.ndteint.2012.11.009.

[24] C. Nucera, F. Lanza, Nonlinear wave propagation in constrained solids subjected to thermal loads, J. Sound Vib. 333 (2014) 541-554. doi:10.1016/j.jsv.2013.09.018.

[25] A. Viswanath, B.P. Chandra, S. Mahadevan, P. Parameswaran, T. Jayakumar, B. Raj, Nondestructive assessment of tensile properties of cold worked AISI type 304 stainless steel using nonlinear ultrasonic technique, J. Mater. Process. Tech. 211 (2011) 538-544. doi:10.1016/j.jmatprotec.2010.11.011.

[26] G. Shui, Y. Wang, F. Gong, Evaluation of plastic damage for metallic materials under 
tensile load using nonlinear longitudinal waves, NDT E Int. 55 (2013) 1-8.

doi:10.1016/j.ndteint.2013.01.001.

[27] J. Kim, L.J. Jacobs, J. Qu, J.W. Littles, Experimental characterization of fatigue damage in a nickel-base superalloy using nonlinear ultrasonic waves, Acoust. Soc. Am. 120 (2006). doi:10.1121/1.2221557.

[28] S. V Walker, J. Kim, J. Qu, L.J. Jacobs, Fatigue damage evaluation in A36 steel using nonlinear Rayleigh surface waves, NDT E Int. 48 (2012) 10-15.

doi:10.1016/j.ndteint.2012.02.002.

[29] D.T. Zeitvogel, K.H. Matlack, J. Kim, L.J. Jacobs, P.M. Singh, J. Qu, Characterization of stress corrosion cracking in carbon steel using nonlinear Rayleigh surface waves, NDT E Int. 62 (2014) 144-152. doi:10.1016/j.ndteint.2013.12.005.

[30] Y.Y. Kim, E.-H. Kim, Effectiveness of the continuous wavelet transform in the analysis of some dispersive elastic waves, J. Acoust. Soc. Am. 110 (2001) 86-94.

[31] S. Thiele, J. Kim, J. Qu, L.J. Jacobs, Air-coupled detection of nonlinear Rayleigh surface waves to assess material nonlinearity, Ultrasonics. 54 (2014) 1470-1475. doi:10.1016/j.ultras.2014.04.020.

[32] L.K. Zarembo, V.A. Krasil'nikov, Nonlinear phenomena in the prop- agation of elastic waves, Sov. Phys. Uspekhi. 13 (1971). doi:10.1088/0741-3335/52/12/124018.

[33] R. Olbrycht, B. Więcek, G. Gralewicz, T. Świątczak, G. Owczarek, Comparison of Fourier and wavelet analyses for defect detection in lock-in and pulse phase thermography, Quant. Infrared Thermogr. J. 4 (2007) 219-232. doi:10.3166/qirt.4.219232.

[34] M. Golmohamadi, H. Badri, A. Ebrahimi, Damage Diagnosis in Bridges Using Wavelet, in: Int. Proc. Comput. Sci. Inf. Technol., 2012: pp. 202-207.

[35] W.L. Bayissa, N. Haritos, S. Thelandersson, Vibration-based structural damage identification using wavelet transform, Mech. Syst. Signal Process. 22 (2008) 1194-1215. doi:10.1016/j.ymssp.2007.11.001.

[36] D. Gabor, Theory of communication, J. Inst. Electr. Eng. III Radio Commun. Eng. 93 
(1946) 429---441.

[37] R.X. Gao, R. Yan, Wavelets, Springer, 2011.

[38] N. Aydin, H.S. Markus, Optimization of processing parameters for the analysis and detection of embolic signals, Eur. J. Ultrasound. 12 (2000) 69-79. doi:10.1016/S09298266(00)00104-X.

[39] L. Cohen, Time-Frequency Distributions --- A Review, Proc. IEEE. 77 (1989) 941-981. doi:10.1109/5.30749.

[40] C.J. Li, J. Ma, Wavelet decomposition of vibrations for detection of bearing-localized defects, NDT E Int. 30 (1997) 143-149.

[41] S. Goedecker, O. V Ivanov, Frequency localization properties of the density matrix and its resulting hypersparsity in a wavelet representation, Phys. Rev. B. 59 (1999) 7270-7273.

[42] B. Basu, V.K. Gupta, Non-Stationary Seismic Response of MDOF Systems by Wavelet Transform, Earthq. Eng. Struct. Dyn. 26 (1997) 1243-1258.

[43] B. Basu, Identification of stiffness degradation in structures using wavelet analysis, Constr. Build. Mater. 19 (2005) 713-721. doi:10.1016/j.conbuildmat.2005.02.018.

[44] L. Gaul, S. Hurlebaus, Identification of the impact location on a plate using wavelets, Mech. Syst. Signal Process. 12 (1998) 783-795.

[45] F. Ykhlef, M. Arezki, A. Guessoum, D. Berkani, A Wavelet Denoising Method to Improve Detection with Ultrasonic Signal, in: IEEE Int. Conf. Ind. Technol., 2004: pp. $1422-1425$.

[46] J. Lin, L. Qu, Feature Extraction Based on Morlet Wavelet and Its Application for Mechanical Fault, Sound Vib. 234 (2000) 135-148. doi:10.1006/jsvi.2000.2864.

[47] S. Mallat, A Wavelet Tour of Signal Processing, 3rd ed., Academic Press, 2008.

[48] J.-P. Antoine, Wavelet Transforms and Their Applications, Phys. Today. 56 (2003) 68-68. doi:10.1063/1.1580056.

[49] M.Z. Silva, R. Gouyon, F. Lepoutre, Hidden corrosion detection in aircraft aluminum structures using laser ultrasonics and wavelet transform signal analysis, Ultrasonics. 41 
(2003) 301-305. doi:10.1016/S0041-624X(02)00455-9.

[50] S.A. Taplidou, L.J. Hadjileontiadis, Nonlinear analysis of wheezes using wavelet bicoherence, Comput. Biol. Med. 37 (2007) 563-570.

doi:10.1016/j.compbiomed.2006.08.007.

[51] A. Teolis, Computational Signal Processing with Wavelets, Springer Science \& Business Media, 2012.

[52] V. Rajagopalan, A. Ray, Symbolic time series analysis via wavelet-based partitioning, Signal Processing. 86 (2006) 3309-3320. doi:10.1016/j.sigpro.2006.01.014.

[53] N. I. H. ImageJ, Image processing and analysis in Java, (2017). https://imagej.nih.gov/ij/.

[54] W. Razvi, S., Li, P., Salama, K., Cantrell Jr, JH., Yost, Nondestructive characterization of aluminum alloys, in: Rev. Prog. Quant. Nondestruct. Eval., Springer, 1987: pp. 14031409.

[55] S. Liu, A.J. Croxford, S.A. Neild, Z. Zhou, Effects of experimental variables on the nonlinear harmonic generation technique, IEEE Trans. Ultrason. Ferroelectr. Freq. Control. 58 (2011) 1442-1451. doi:10.1109/TUFFC.2011.1963.

[56] R.P. Agarwal, P.J.Y. Wong, Error Inequalities in Polynomial Interpolation and Their Applications, Springer Netherlands, 1993.

[57] L.D. Landau, E.M. Lifshitz, Theory of elasticity: Transl. from the Russian by JB Sykes and WH Reid, (1975) 195. 